\title{
Higher-order QCD corrections to vector boson production at hadron colliders.
}

\author{
Giancarlo Ferrera \\ Dipartimento di Fisica, Università di Firenze \& INFN Sez. di Firenze, I-50019 Sesto Fiorentino, \\ Florence, Italy \\ E-mail: ferreradfi.infn.it
}

\begin{abstract}
We present two recent results on higher-order QCD corrections to the production of vector bosons in hadron collisions.

We discuss the resummation of logarithmic-enhanced QCD corrections at small values of $q_{T}$ and the matching procedure to consistently combine resummation with the fixed-order perturbative result at intermediate and large $q_{T}$. We study the perturbative uncertainty of the results and we compare our prediction with Tevatron data for $Z$ bosons production.

Moreover we discuss a fully exclusive calculation up to next-to-next-to-leading order (NNLO) in QCD perturbation theory. The calculation is implemented in a parton level Monte Carlo program which allows the user to apply arbitrary kinematical cuts on the final-states and to compute the corresponding distributions in the form of bin histograms.
\end{abstract}

European Physical Society Europhysics Conference on High Energy Physics, EPS-HEP 2009, July 16 - 222009

Krakow, Poland 


\section{Transverse-momentum resummation}

We are interested in the high-energy collisions of the hadrons $h_{1}$ and $h_{2}$ which produce a vector boson $V$ (which decays into the lepton pair $l_{1}, l_{2}$ ) plus an arbitrary and undetected final state $X$

$$
h_{1}+h_{2} \rightarrow V\left(M, q_{T}\right)+X \rightarrow l_{1}+l_{2}+X,
$$

where $q_{T}$ and $M$ are respectively the transverse momentum and the invariant mass of the vector boson.

We consider the transverse-momentum distribution and we identify two different kinematical regions. In the region where $q_{T} \sim m_{V}, m_{V}$ being the mass of the vector boson $\left(m_{V}=m_{W}, m_{Z}\right)$, the QCD perturbative series is controlled by a small expansion parameter, $\alpha_{S}\left(m_{V}\right)$. In this region the fixed-order QCD calculations, known up to next-to-leading order (i.e. $\mathscr{O}\left(\alpha_{S}^{2}\right)$ ) [1], are theoretically justified. In the small- $q_{T}$ region $\left(q_{T} \ll m_{V}\right)$, the convergence of the fixed-order perturbative expansion is spoiled by the presence of powers of large logarithmic terms, $\alpha_{S}^{n} \ln ^{m}\left(m_{V}^{2} / q_{T}^{2}\right)$. In order to obtain reliable predictions in such region an all order resummation of these terms is mandatory.

The $q_{T}$ resummation is performed at the level of the partonic cross section, which is decomposed in two terms: $d \hat{\sigma}^{V} / d q_{T}^{2}=d \hat{\sigma}^{V \text { (res.) }} / d q_{T}^{2}+d \hat{\sigma}^{V \text { (fin.) }} / d q_{T}^{2}$ [2,3]. The term $d \hat{\sigma}^{V \text { (res.) }}$ contains all the logarithmically enhanced contributions (at small $q_{T}$ ) we have to resum while the term $d \hat{\sigma}^{V(\text { fin.) }}$ is free of such contributions and can be evaluated at fixed order in perturbation theory.

The resummation procedure is performed in the impact-parameter space through a FourierBessel transform

$$
\frac{d \hat{\sigma}^{V(\text { res. })}}{d q_{T}^{2}}\left(q_{T}, M, \hat{s}, \alpha_{S}\right)=\hat{\sigma}_{L O}^{V}(M) \frac{M^{2}}{\hat{s}} \int_{0}^{\infty} d b \frac{b}{2} J_{0}\left(b q_{T}\right) \mathscr{W}^{V}\left(b, M, \hat{s}, \alpha_{S}\right),
$$

where the impact parameter $b$ is the conjugate variable with respect to $q_{T}, J_{0}(x)$ is the 0 -order Bessel function and $\hat{\sigma}_{L O}^{V}$ is the Born partonic cross section. We can now write the partonic resummed component $\mathscr{W}^{V}\left(b, M, \hat{s}, \alpha_{S}\right)$ in the exponential form by considering its $N$-moments with respect to the variable $z=M^{2} / \hat{s}$ at fixed $M$

$$
\mathscr{W}_{N}^{V}\left(b, M, \alpha_{S}\right)=\mathscr{H}_{N}^{V}\left(\alpha_{S}\right) \times \exp \left\{\mathscr{G}_{N}\left(\alpha_{S}, L\right)\right\} \text {, with } L=\ln \left(Q^{2} b^{2} / b_{0}^{2}\right), b_{0}=2 e^{-\gamma_{E}} .
$$

We have introduced in the above formula the scale $Q \sim M \sim m_{V}$, the so called resummation scale, which has a role analogous to the factorization and renormalization scales: variations of $Q$ around $m_{V}$ can be used to estimate the size of higher-order logarithmic contributions that are not explicitly resummed in a given calculation.

The process dependent function $\mathscr{H}_{N}^{V}$ includes all the perturbative terms that behave as constants as $q_{T} \rightarrow 0$. It can thus be expanded in powers of $\alpha_{S}=\alpha_{S}\left(\mu_{R}^{2}\right)$ :

$$
\mathscr{H}_{N}^{V}\left(\alpha_{S}\right)=\left[1+\frac{\alpha_{S}}{\pi} \mathscr{H}_{N}^{V(1)}\left(\frac{\alpha_{S}}{\pi}\right)^{2} \mathscr{H}_{N}^{V(2)}+\ldots\right]
$$

The universal exponent $\mathscr{G}_{N}$ resums all the terms that order-by-order in $\alpha_{S}$ are logarithmically divergent.

Finally the finite component has to be evaluated starting from the usual fixed-order perturbative truncation of the partonic cross section and subtracting the expansion of the resummed part at the 
same perturbative order: $\left[d \hat{\sigma}^{V \text { (fin.) }} / d q_{T}^{2}\right]_{\text {f.o. }}=\left[d \hat{\sigma}^{V} / d q_{T}^{2}\right]_{\text {f.o. }}-\left[d \hat{\sigma}^{V(\text { res. })} / d q_{T}^{2}\right]_{\text {f.o. }}$. This matching procedure is important to achieve uniform theoretical accuracy over the entire range of transverse momenta.

To perform a resummation at next-to-next-to-leading logarithmic order, the knowledge of the coefficient $\mathscr{H}_{N}^{V(2)}$ is necessary. Since this coefficient has been computed only recently [4], here we limit ourselves to presenting results up to next-to-leading logarithmic accuracy matched with the leading fixed-order result (NLL+LO).

In Fig. 1 we compare our NLL+LO resummed spectrum [3] (with different values of the factorization, renormalization and resummation scale) with the Tevatron D0 Run II data [5]. We find that the scale uncertainty is about $\pm 12-15 \%$ from the region of the peak up to the intermediate $q_{T}$ region $\left(q_{T} \sim 20 \mathrm{GeV}\right)$, and it is dominated by the resummation-scale uncertainty. Taking into account the scale uncertainty, we see that the resummed curve agrees reasonably well with the experimental points ${ }^{1}$. We expect a sensible reduction of the scale dependence once the complete NNLL+NLO calculation is available.
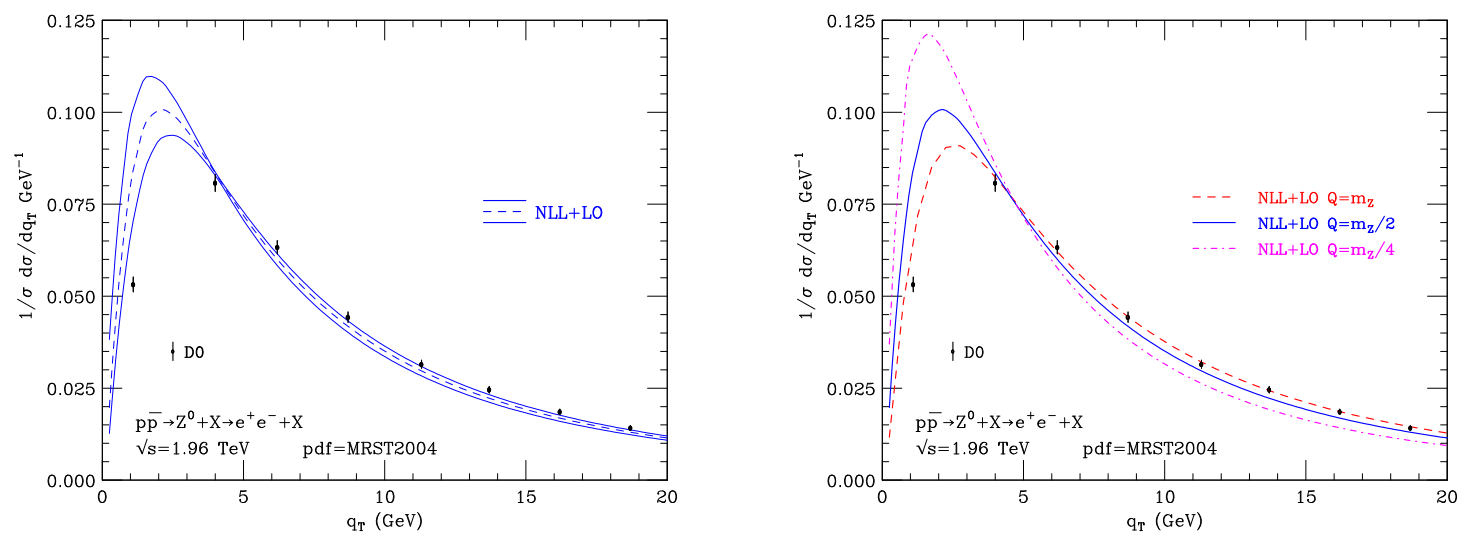

Figure 1: The $q_{T}$-spectrum of the Drell-Yan $e^{+} e^{-}$pairs produced in $p \bar{p}$ collisions at the Tevatron Run II [5]. Theoretical results are shown at NLL+LO, including scale variations. Left side: $m_{Z} / 2 \leq \mu_{F}, \mu_{R} \leq 2 m_{Z}$, with the constraint $1 / 2 \leq \mu_{F} / \mu_{R} \leq 2$. Right side: $m_{Z} / 4 \leq Q \leq m_{Z}$

\section{Fully exclusive NNLO calculation}

We now consider a generic observable $d \hat{\sigma}^{V}$ for the process in Eq. 1.1. We present a computation of the next-to-next-to-leading order (NNLO) QCD radiative corrections for such observable with arbitrary (though infrared safe) kinematical cuts on the final-state [4]. Provided the observable is sufficiently inclusive over the small- $q_{T}$ region, resummation is not necessary and fixed-order perturbation theory can be used.

Following Ref. [6], we observe that, at LO, the transverse momentum $q_{T}$ of $V$ is exactly zero. This means that if $q_{T} \neq 0$ the $(\mathrm{N}) \mathrm{NLO}$ contributions is given by the (N)LO contribution to the final state $V+j e t(s):\left.d \hat{\sigma}_{(N) N L O}^{V}\right|_{q_{T} \neq 0}=d \hat{\sigma}_{(N) L O}^{V+\text { jets }}$. We compute $d \hat{\sigma}_{N L O}^{V+\text { jets }}$ by using the subtraction

\footnotetext{
${ }^{1}$ We note that in Fig. 1 the theoretical results are obtained in a pure perturbative framework, without introducing any models of non-perturbative contributions. These contributions can be relevant in the $q_{T}$ region below the peak.
} 
method at NLO and we treat the remaining NNLO singularities at $q_{T}=0$ by the additional subtraction of a counter-term constructed by exploiting the universality of the logarithmically-enhanced contributions to the $q_{T}$ distribution (see Eq. 1.3)

$$
d \hat{\sigma}_{(N) N L O}^{V}=\mathscr{H}_{(N) N L O}^{V} \otimes d \hat{\sigma}_{L O}^{V}+\left[d \hat{\sigma}_{(N) L O}^{V+\mathrm{jets}}-d \hat{\sigma}_{(N) L O}^{C T}\right],
$$

where $\mathscr{H}_{(N) N L O}^{V}$ is the process dependent coefficient function of Eq. 1.4.

We have encoded our NNLO computation in a parton level Monte Carlo event generator. The calculation includes finite-width effects, the $\gamma-Z$ interference, the leptonic decay of the vector bosons and the corresponding spin correlations ${ }^{2}$. Our numerical code is particularly suitable for the computation of distributions in the form of bin histograms, as shown the illustrative numerical results presented in Fig. 2 .
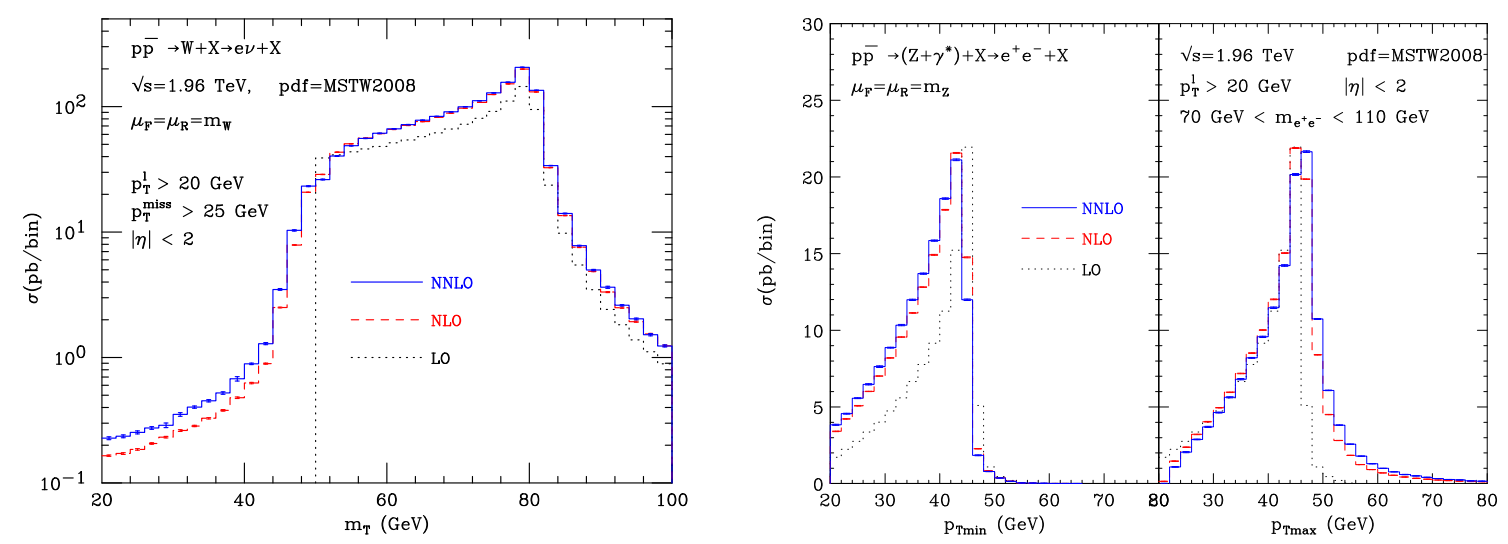

Figure 2: Left side: transverse mass distribution for $W$ production at the Tevatron. Right side: distributions in $p_{T \min }$ and $p_{T \max }$ for the $Z$ signal at the Tevatron.

\section{References}

[1] P. B. Arnold and M. H. Reno, Nucl. Phys. B 319, 37 (1989) [Erratum-ibid. B 330, 284 (1990)]; R. J. Gonsalves, J. Pawlowski and C. F. Wai, Phys. Rev. D 40, 2245 (1989).

[2] G. Bozzi, S. Catani, D. de Florian and M. Grazzini, Nucl. Phys. B 737 (2006) 73, Phys. Lett. B 564 (2003) 65 [arXiv:hep-ph/0302104]; Nucl. Phys. B 791 (2008) 1 [arXiv:0705.3887 [hep-ph]].

[3] G. Bozzi, S. Catani, G. Ferrera, D. de Florian and M. Grazzini, Nucl. Phys. B 815 (2009) 174 [arXiv:0812.2862 [hep-ph]].

[4] S. Catani, L. Cieri, G. Ferrera, D. de Florian and M. Grazzini, Phys. Rev. Lett. 103 (2009) 082001 [arXiv:0903.2120 [hep-ph]].

[5] V. M. Abazov et al. [D0 Coll.], Phys. Rev. Lett. 100 (2008) 102002 [arXiv:0712.0803 [hep-ex]].

[6] S. Catani and M. Grazzini, Phys. Rev. Lett. 98 (2007) 222002 [arXiv:hep-ph/0703012].

[7] K. Melnikov and F. Petriello, Phys. Rev. Lett. 96 (2006) 231803 [arXiv:hep-ph/0603182].

\footnotetext{
${ }^{2}$ In the quantitative studies that we have carried out, our computation gives results in numerical agreement with the calculation, performed with a different method, presented in Ref. [7].
} 